\title{
Outcome After Partial Ileal Bypass for the Treatment of Ileocecal Intussusception in 6 Horses
}

\author{
Nicola Pilati*, Rodolfo Gialletti, Sara Nannarone and Marco Pepe \\ Department of Medicina Veterinaria, University of Perugia, Italy
}

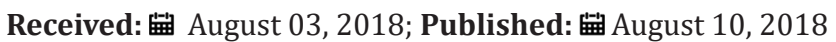

*Corresponding author: Nicola Pilati, Dipartimento di Medicina 10 Veterinaria, Via San Costanzo, 06123 Perugia, Italy

\begin{abstract}
Ileocecal intussusceptions represent a not rare cause of colic in horses; different technique is described to surgically treat this lesion and the report point its attention to the partial ileal bypass focusing on the long term follow-up and possible complications of this procedure. Six horses presented for signs of abdominal pain underwent a clinical, ultrasonographic and rectal examination where a presumptive diagnosis of ileocecal intussusception was made in all but one patient. All horses underwent exploratory laparotomy where a definitive diagnosis of ileocecal intussusception was made; a jejuno-cecostomy with no resection of the ileum (partial ileal bypass) and no reduction of the lesion was performed in all the patients. The follow up revealed no long-term complications in all the horses.
\end{abstract}

Keywords: Colic; Horse; Intussusception; Laparotomy

\section{Introduction}

Colic can be considered as one of the leading causes of death in horses, and although the majority of cases can be managed with a medical approach, a small percentage requires surgery to avoid a fatal outcome [1]. Several studies in recent decades have investigated the survival rates and complications after colic surgery, focusing attention on the variables that may affect the outcome [24]. According to one study [2], surgical lesions affecting the ileum, or the ileocecal junction were found in $6.6 \%$ of a group of 300 horses; ileocecal intussusception occurred in $1.3 \%$ of the entire sample. This lesion often occurs in young horses, and the initial clinical signs vary from an acute colic syndrome to chronic weight loss and poor body condition. Ileocecal intussusceptions are not always easy to diagnose on clinical examination because of the position of this organ in the abdomen. Surgical treatment of this part of the small intestine can be challenging because of the inability to exteriorize the most caudal part of the ileum, close to the ileocecal junction. Different surgical approaches can be considered for the treatment of ileocecal intussusceptions. English language literature includes studies describing manual reduction of the lesion or resection of the involved segments and a subsequent complete bypass of the ileum, jejunocecostomy with transection of the ileum $[2,5,6]$.

The aim of this study was to investigate whether jejunocecostomy without resection of the ileum and reduction of the lesion is a reasonable technique for surgical treatment of ileocecal intussusception and possible long term compications. The report describes the diagnostic trial, the surgical treatment and the short- and longterm follow-up of a group of horses that underwent a partial ileal bypass leaving the intussusception in situ.

\section{Case History}

The medical records of all horses admitted for signs of colic to the Veterinary Teaching Hospital of the University of Perugia that underwent exploratory laparotomy from January 2011 to December 2013 were reviewed retrospectively. Horses diagnosed with an ileocecal intussusception that underwent colic surgery in which a jejunocecostomy with no resection of the ileum (partial ileal bypass) was performed were identified. Data on age and sex obtained from the clinical examination at admission and any complications occurred during hospitalization were recorded. A telephone questionnaire was used to collect long-term follow-up information from owners $\backslash$ trainers, with emphasis on any possible complications, such as episodes of colic, poor body condition, capricious appetite, and the level of athletic activity. Six horses met the inclusion criteria. Their age ranged from 1 to 18 years (mean 4.33, median 1.25 years), there were two fillies and four were intact males with a breed distribution (5 Thoroughbred, 1 Arabian) not reflective of the general hospital population.

The history revealed that the duration of abdominal pain before admission was less than 6 hours in one horse, between 6 and 24 hours in two and more than 24 hours in three patients. Medical treatments administered by the referring veterinarians before referral included fluid therapy in five horses, non-steroidal antiinflammatories (NSAIDs) in six horses, mineral oil via nasogastric tube in one horse and xylazine hydrochloride in four horses. The physical examination at admission revealed that one patient had signs of severe abdominal discomfort while the other five horses had mild to moderate abdominal pain. Rectal temperature 
ranged from 38.0 to $39.2^{\circ} \mathrm{C}$ (mean $38.5^{\circ} \mathrm{C}$ ) and the heart rate was recorded at 40 to 60 beats $\backslash$ minute (mean 50.3 beats $\backslash$ minute). Mucous membranes were hyperemic in five horses and brick red in the other. Borborygmi were depressed in all the patients. No net nasogastric reflux was obtained in any of the patients. The clinical examination was followed by abdominal ultrasound, using a 3.5 $\mathrm{MHz}$ convex probe, and rectal examination in all cases; transrectal ultrasound examination using a $5 \mathrm{MHz}$ microconvex probe was performed in two cases.

A preoperative diagnosis of ileal intussusception was made in five of the six horses before surgery: with transabdominal ultrasound in two horses, with rectal palpation in one and with transrectal ultrasound in two; only non-specific signs of small intestinal disease, including small intestinal distension and loops, were found on ultrasound examination of the remaining horse. The clinical laboratory findings at admission included a packed cell volume that ranged from $36 \%$ to $40 \%$ (mean 39\%) and total protein concentration that ranged from 5 to $7 \mathrm{~g} / \mathrm{dl}$ (mean $5.7 \mathrm{~g} /$ dl). Peritoneal fluid analysis was not performed in any of the cases.

\section{Treatment}

All horses underwent exploratory celiotomy because of a preoperative diagnosis of ileocecal intussusception or signs of small intestinal disease. Surgical preparation, anesthesia and postoperative care were similar for all the patients included in the study. Broad-spectrum antimicrobial therapy with gentamicin (6.6 $\mathrm{mg} / \mathrm{kg}$ bodyweight[btw] intravenously[i.v.]) and potassium penicillin $(22,000 \mathrm{IU} / \mathrm{kg}$ bwt intramuscularly[i.m.]), together with flunixin meglumine at $1 \mathrm{mg} / \mathrm{kg}$ bwt i.v., were administered as pre-operative medications. The horses were sedated with xylazine hydrochloride (1.1 mg/kg bwt i.v.) and butorphanol tartrate $(0.02 \mathrm{mg} / \mathrm{kg}$ bwt i.v.). General anesthesia was induced with ketamine (2-2.2 mg/kg bwt i.v.) and diazepam $(0.04 \mathrm{mg} / \mathrm{kg}$ bwt i.v.) and maintained with isoflurane in $100 \%$ oxygen using a semi-closed anesthetic circuit. After midline celiotomy, the abdomen was explored, and the lesion of the ileum identified: an attempt to reduce the lesion was not performed in all the patients. The surgical technique used was always a side-to-side two-layer jejunocecostomy where the selected area of the jejunum was opposed to the cecum between the dorsal and the medial band of this organ on its proximal third. The small intestine was directed in an apex-base orientation. The authors used a hand-sewn two-layer

\section{Technique}

The selected area of the cecum was isolated with two curved Doyen intestinal forceps to create a pouch and to avoid contamination during the procedure, and the antimesenteric margin of the jejunum was opposed to it. Using a serosubmucosal simple continuous pattern, interrupted at the two poles, the two segments of intestine were connected at the far side and the seromuscular layer of the jejunum and the cecum were incised for an approximate length of 10-12 cm. A serosubmucosal simple continuous pattern was then performed in the near side and the mucosa gradually incised to create the stoma. The first row was then oversewn using a Cushing continuous pattern. The suture material used was $2 \backslash 0$ USP, 3 metric Poliglecaprone with a taper needle. The stoma was reported to be approximately $10-12 \mathrm{~cm}$ long. The mean surgical time was 77.5 minutes (range 50-100 minutes), considering the time lapsed between the celiotomy incision and the skin closure. All the horses recovered from anesthesia with no complications and were closely monitored.

They received appropriate i.v. fluid therapy depending on their hydration status, and flunixin meglumine ( $1 \mathrm{mg} / \mathrm{kg}$ bwt i.v.) every 12 hours for two days and then every 24 hours for three more days. Broad-spectrum antimicrobial therapy was administered for five days postoperatively (gentamicin $6.6 \mathrm{mg} / \mathrm{kg}$ bwt i.v. once a day; potassium penicillin $22,000 \mathrm{IU} / \mathrm{kg}$ bwt i.m. twice a day). Four horses presented minor postoperative complications during hospitalization. Two patients had a moderate infection of the surgical incision with purulent discharge and involvement of the cutaneous and subcutaneous structures; this was treated with local antiseptic solutions and resolved before discharge from the clinic. One horse had diarrhea from day 2 to day 5 postoperatively and had signs of abdominal discomfort on day 10. On clinical, ultrasonographic and rectal examinations a left dorsal displacement of the colon was diagnosed and successfully treated medically. This horse also presented an infection of the surgical incision with involvement of the muscular layers; this was treated with topical solutions and abdominal bandaging and resolved clinically on day 30 postoperatively.

One horse presented postoperative fever at days 3 and 4 for no apparent clinical reason: it turned out seropositive to Theileria equi $(\operatorname{IgG}>1 / 640)$ and Babesia caballi $(\operatorname{IgG}>1 / 320)$ and therefore a diagnosis of piroplasmosis was made. The patient was treated with Imidocarb (17 mg/kg i.m.). The duration of hospitalization was recorded in all horses and ranged from 9 to 25 (mean16.6 days). Follow-up information was obtained for all the patients by telephone interview with the owner $\backslash$ trainer $\backslash$ referring veterinarian and ranged from 12 to 30 months (mean 22.3 months). None of the horses had long-term complications, good body condition score was reported and all of them were used as intended.

\section{Discussion}

This case series describes the use of a partial bypass of the ileum with no reduction of the lesion for surgical treatment of ileocecal intussusception and focuses on the short- and long-term follow-up of the horses included in the study. The ileum is the most caudal part of the small intestine, with an approximate length of 70 $\mathrm{cm}$ in the horse. It arises from the jejunum in the left flank, crosses to the right flank at the fourth lumbar vertebra and ends at the ileal orifice of the lesser curvature of the base of the caecum. Therefore, the ileocecal orifice is situated in the right dorsal quadrant of the abdomen and, because of the short mesenteric attachment of the cecum, it is almost immobile [7]. The position of this part of the small intestine in the abdomen can make the preoperative diagnosis of occluding lesions of the ileum challenging. One author [8] was able to detect impaction of the ileum in only 11 of the 28 horses included in his study on clinical examination by rectal palpation, another [9] was able to make a preoperative diagnosis of ileocecal intussusception in 16 of 37 horses, 13 by rectal palpation and 3 by transabdominal ultrasound while Edwards [5] diagnosed only one 
of three ileocecal intussusceptions by rectal examination. In recent years, ultrasound examination of the abdomen has tremendously improved our ability to make a preoperative diagnosis [10], this is due to improvements both in the technique and in the technology of ultrasound machines.

It is the author's opinion that ultrasound examination of the abdomen can give diagnostic details not possible to obtain by other means and this technique has a primary role in the detection of ileal diseases, especially ileocecal intussusception. The complementary use of a transabdominal and a transrectal approach [11,12] can provide important information when an ileal disease is present, although this contradicts statements made by other authors [9]. Transabdominal ultrasound examination combined with the transrectal approach allowed us to make a preoperative diagnosis of ileal intussusception in four cases where rectal examination was unremarkable. This allows us to state that the systematic use of a combination of abdominal and rectal ultrasound examination of the patient with colic can allow the clinician to make a preoperative diagnosis of ileal disease, although the size, the temperament of the horse or the degree of abdominal pain may limit the use of the transrectal approach. Different surgical techniques to treat ileocecal intussusception are described in the literature. Manual reduction of the lesion can be considered an option but, because the ileum may appear healthy macroscopically while being functionally impaired, resection of the ileum and a side-to-side jejunocecal anastomosis has been considered the best way to treat the lesion by one author [5].

However, this technique has been associated with postoperative complications and poorer survival than other anastomotic techniques. One author [2] stated that only approximately $68 \%$ of patients treated by jejunocecostomy with resection of the ileum survived in the short term, the second lower percentage analyzing the short-term survival after different kind of anastomosis of the small and large intestine, and this was in agreement with the results of other authors $[4,13]$. This was attributed to mechanical complications caused by transitions between intestinal segments of dissimilar function, to the inability to resect all the affected ileum, and to the high risk of adhesion formation on the ileal stump [6]. The surgical technique used in this case series was a jejunocecostomy with no resection of the affected ileum and no reduction of the lesion. This technique has been described by different authors $[6,7]$ but in the last two decades only a few reports have described its use and the outcome after diagnosis of ileal obstruction.

One author [9] described the short-term follow-up of a group of 37 horses affected by ileocecal intussusception that were treated with a partial bypass of the ileum and no reduction of the lesion, and this is so far the largest study in the English language literature that describes short-term follow-up of this technique. The results were similar to those of our case series in that the patients had no short-term complications, and it agrees with the results of another author who used the same surgical approach to treat other types of ileal obstruction [14]. Reduction of the intussusceptum is not mandatory: in agreement with Bell [9], it is the author's opinion that the likelihood of bleeding from the intussusceptum or septic peritonitis caused by leakage or rupture of the lesion is not high, because the compromised bowel remains walled off from the peritoneal cavity. No long-term complications were found in this case series using this technique. This means that leaving the intussusception in situ is a reasonable choice, rather than reducing the lesion and resecting the involved ileum, considering the high percentage of postoperative complications associated with the latter technique, as described by different authors $[2,4,15]$. Some postoperative complications have been described for bypass procedures of the ileocecal valve, due especially to the fact that the stoma can shrink down and occlude the lumen of the anastomosis (Mair et al. 2000)16 or the fact that leaving a long intussusceptum in situ can impinge on the cecocolic orifice and create an obstruction to the normal flow of the ingesta between these two tracts of the intestine [16].

The authors did not encounter such complications in the cases included in this study, but the intussusceptions were always shorter than $15 \mathrm{~cm}$ and we suspect that to treat in a similar fashion intussusceptions longer than $20 \mathrm{~cm}$ the clinician may risk occlusion of the cecocolic orifice so other techniques should be used. The suture pattern used was a two-layer side-to-side hand-sewn jejunocecostomy. Other techniques described include a stapled or hand-sewn single-layer semiclosed jejunocecal sideto-side anastomosis: the first can be considered relatively less time consuming but has a higher risk of complications if not over sewn, and the cost of stapling made the surgeon in this case series decide to use a hand-sewn technique. The latter method was first described by one author [14], who successfully treated six cases of ileal impaction with a partial ileal bypass. It can be considered, in the authors' opinion, a valuable suture pattern but because it was only recently published it was not taken into consideration by the surgeon in this case series. In conclusion, the partial ileal bypass with no resection of the ileum and no reduction of the intussusception can be considered an efficacious surgical treatment for ileocecal intussusceptions. According to the results of this case series and together with data in the literature6, this technique gives good short- and long-term results. It is however important to note that larger numbers of cases should be studied to better evaluate the long-term follow-up after this procedure.

\section{References}

1. Hillyer MH, Taylor FGR, French NP (2001) A cross-sectional study of colic in horses on Thoroughbred training premises in the British Isles in 1997. Equine Veterinary Journal 33(4): 380-385.

2. Mair TS, Smith LJ (2005) Survival and complication rates in 300 horses undergoing surgical treatment of colic. Part 1: short-term survival following a single laparotomy. Equine veterinary journal 37 (4): 296-302.

3. Mair TS, Smith LJ (2005) Survival and complication rates in 300 horses undergoing surgical treatment of colic. Part 3: Long-term complications and survival. Equine veterinary journal 37(4): 310-314.

4. Freeman E, Hammock P, Baker GJ, Goetz T, Foreman JH, et al. (2000) Short-and long-term survival and prevalence of postoperative ileus after small intestinal surgery in the horse. Equine Veterinary Journal 32: 4251.

5. Edwards GB (1986) Surgical management of intussusception in the horse. Equine veterinary journal 18(4): 313-321. 
6. Ford TS, Freeman E, Ross MW, Richardson DW, Martin BB, et al. (1990) Ileocecal intussusception in horses: 26 cases (1981-1988). Journal of the American Veterinary Medical Association 196(1): 121-126.

7. Edwards GB (1981) Obstruction of the ileum in the horse: a report of 27 clinical cases. Equine veterinary journal 13(3): 158-166.

8. Hanson R, Wright JC, Schumacher J, Baird AN, Humburg J, et al. (1998) Surgical reduction of ileal impactions in the horse: 28 cases. Veterinary Surgery 27(6): 555-560.

9. Bell RJW, Textor JA (2010) Caecal intussusceptions in horses: a New Zealand perspective. Australian veterinary journal 88(7): 272-276.

10. Beccati F, Pepe M, Gialletti R, Cercone M, Bazzica C, et al. (2011) Is there a statistical correlation between ultrasonographic findings and definitive diagnosis in horses with acute abdominal pain? Equine Veterinary Journal 39: 98-105.

11. Slack J (2013) Abdominal Sonographic Ultrasound. In: Southwood LL Pratical Guide to Equine Colic. Pp. 116-48.

12. Freeman SL (2003) Diagnostic ultrasonography of the mature equine abdomen. Equine Veterinary Education 15(6): 319-330.

ISSN: 2574-1241

DOI: 10.26717/BJSTR.2018.07.001559

Nicola Pilati. Biomed J Sci \& Tech Res

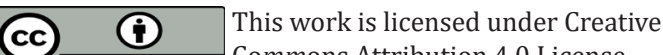

Submission Link: https://biomedres.us/submit-manuscript.php
13. Phillips TJ, Walmsley JP (1993) Retrospective analysis of the results of 151 exploratory laparotomies in horses with gastrointestinal disease. Equine veterinary journal 25(5): 427-431.

14. Gandini M (2010) Handsewn Semiclosed Single-Layer Jejunocecal SideTo-Side Anastomosis in the Horse. Veterinary Surgery 39(6): 771-775.

15. Blandon BM, Hilyer MH (2000) Effect of extensive ileal resection with a large resulting mesenteric defect and stapled ileal stump in horses with a jejunocaecostomyl a comparison with other anastomotic techniques. Equine Veterinary Journal Supplement 32: 52-58.

16. Schumacher J, Hanrahan L (1987) Ileocecocolic intussusception as a sequel to jejunocecostomy in a mare. Journal of the American Veterinary Medical Association 190(3): 303-304.

17. Mair TS, Lucke VM (2000) Ileal muscular hypertrophy and rupture in a pony three years after surgery for ileocaecal intussusception. Veterinary Record 146(16): 472-473.

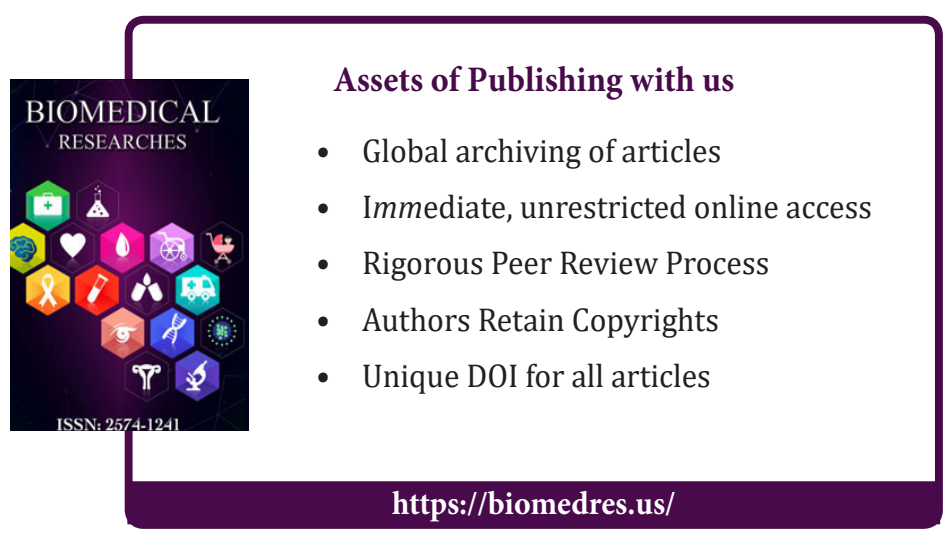

